\title{
An optimized reference current detection method for active power filter
}

\author{
Dongdong Chen ${ }^{\text {a) }}$ and Guozhu Chen \\ College of Electrical Engineering, Zhejiang University, \\ Hangzhou, 310027, China
}

a)3090104956@zju.edu.cn

\begin{abstract}
An optimized reference current detection method based on synchronous reference frame transformation and sliding discrete Fourier transform (SDFT) is proposed. The proposed method can be used to extract characteristic harmonic currents as the reference current of system from the load currents by fewer sampling points, meanwhile, the numbers of harmonics need to be calculated will reduce to half of traditional method, it means that the proposed method can reduce the computational effort and easy to engineering implementation, while achieving better dynamic performance compared with other methods. The results of simulation and experiment show the validity of this method.
\end{abstract}

Keywords: active power filter (APF), reference current detection, synchronous reference frame, SDFT

Classification: Energy harvesting devices, circuits and modules

\section{References}

[1] Z. Luo, et al:: "Analysis and control of a reduced switch hybrid active power filter," IET Power Electron. 9 (2016) 1416 (DOI: 10.1049/iet-pel.2015.0027).

[2] S. Luetkemeier, et al.: "A $200 \mathrm{mV} 32 \mathrm{~b}$ subthreshold processor with adaptive supply voltage control,” ISSCC Dig. Tech. Papers (2012) 484 (DOI: 10.1109/ ISSCC.2012.6177101).

[3] Y. F. Wang, et al.: "Three-phase cascaded delayed signal cancellation PLL for fast selective harmonic detection," IEEE Trans. Ind. Electron. 60 (2013) 1452 (DOI: 10.1109/TIE.2011.2162715).

[4] H. Li, et al:: "A novel time-domain current-detection algorithm for shunt active power filters," IEEE Trans. Power Syst. 20 (2005) 644 (DOI: 10.1109/TPWRS. 2005.846215).

[5] C. Gui, et al.: "An improved adaptive minimum dc-link voltage controller," IEICE Electron. Express 13 (2016) 20160429 (DOI: 10.1587/elex.13. 20160429).

[6] N. A. Rahim, et al:: "DSP-based flexible digital hysteresis in switched capacitor active power filter," IEICE Electron. Express 7 (2010) 621 (DOI: 10. 1587/elex.7.621).

[7] H. Wang, et al.: "Investigation on a new algorithm for instantaneous reactive and harmonic currents detection applied to intensive nonlinear loads," IEEE Trans. Power Delivery 22 (2006) 2312 (DOI: 10.1109/TPWRD.2007.905379). 
1109/TPEL.2010.2103368).

[9] D. Tian, et al.: "A selective harmonic optimization method for STATCOM in steady state based on the sliding DFT," 2015 IEEE International Conference on Systems, Man, and Cybernetics, Kowloon (2015) 2049 (DOI: 10.1109/SMC. 2015.358).

[10] S. Chandrasekaran and K. Ragavan: "Adaptive sampling period adjusted sliding DFT for synchronous reference frame PLL," 2014 IEEE International Conference on Power Electronics, Drives and Energy Systems (PEDES), Mumbai (2014) 1 (DOI: 10.1109/PEDES.2014.7041974).

[11] K. Sozanski: Power Theories for Improved Power Quality (Springer London, 2012).

\section{Introduction}

Active power filter (APF) is a new kind of the power electronic device used to effectively suppression harmonic current generated by the nonlinear loads [1]. Extracting the Harmonics which need to be compensated as reference current determine the steady state compensation accuracy and dynamic response capability of the active power filter. The traditional method based on the instantaneous reactive power theory $[2,3,4,5,6]$, when the load steps dynamically, the DClink voltage may jump greatly. The SDFT method based on the abc coordinate system has been widely used in harmonic refrence current calculation $[6,7,8,9]$, but it has an inherent latency of 1 fundamental grid cycle [10,11], which makes the system dynamic performance becomes poor, reducing the compensation accuracy.

The shunt APF structure and control block is shown in Fig. 1.

A three-phase shunt APF is connected with the grid through a LCL filter, and DC-link capacitor is $C_{d c}$. In the control part, $I_{r}$ is the reference current extract from load current $I_{l h}$ for the current loop control; and the output of current loop to generate the PWM through the PWM block.

In this paper, an optimized reference current detection method is proposed, which is based on SDFT harmonics detection method in the synchronous reference frame.

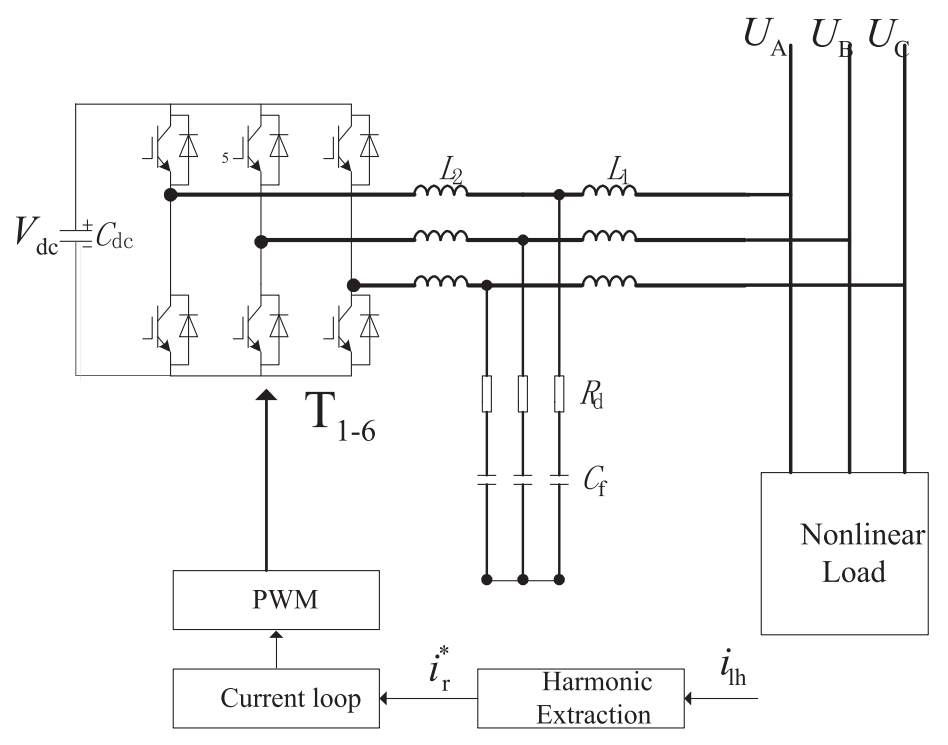

Fig. 1. System structure of the shunt APF 


\section{Implementation}

\subsection{Harmonics in synchronous reference frame}

Generally speaking, a balanced three-phase system only contains $(6 n \pm 1)$ th $(\mathrm{n}=$ $0,1,2 \ldots$ ) harmonics because many power conversion processes (i.e., diode rectifier loads) involved in industrial applications produce harmonic components at these frequencies.

$$
i_{\text {habc }}(t)=\left\{\begin{array}{l}
\sum_{\substack{n=6 k-1 \\
k=1,2,3, \ldots}}^{\infty} \sqrt{2} I_{n} \sin \left(n \omega_{0} t+\phi_{h n}\right) \\
+\sum_{\substack{n=6 k+1 \\
k=1,2,3, \ldots}}^{\infty} \sqrt{2} I_{n} \sin \left(n \omega_{0} t+\phi_{h p}\right) \\
\sum_{\substack{n=6 k-1 \\
k=1,2,3, \ldots}}^{\infty} \sqrt{2} I_{n} \sin \left(n \omega_{0} t+\phi_{h n}+\frac{2 \pi}{3}\right) \\
+\sum_{\substack{n=6 k+1 \\
k=1,2,3, \ldots}}^{\infty} \sqrt{2} I_{n} \sin \left(n \omega_{0} t+\phi_{h p}-\frac{2 \pi}{3}\right) \\
\sum_{\substack{n=6 k-1 \\
k=1,2,3, \ldots}}^{\infty} \sqrt{2} I_{n} \sin \left(n \omega_{0} t+\phi_{h n}-\frac{2 \pi}{3}\right) \\
+\sum_{\substack{n=6 k+1 \\
k=1,2,3, \ldots}}^{\infty} \sqrt{2} I_{n} \sin \left(n \omega_{0} t+\phi_{h p}+\frac{2 \pi}{3}\right)
\end{array}\right.
$$

In positive synchronous reference frame:

$$
\begin{aligned}
{\left[\begin{array}{c}
i_{h d} \\
i_{h q}
\end{array}\right]=} & \sum_{\substack{n=6 k-1 \\
k=1,2,3, \ldots}}^{\infty} \sqrt{3} I_{n}\left[\begin{array}{c}
\cos \left[(n+1) \omega_{0} t+\phi_{h n}\right] \\
\sin \left[(n+1) \omega_{0} t+\phi_{h n}\right]
\end{array}\right] \\
& +\sum_{\substack{n=6 k+1 \\
k=1,2,3, \ldots}}^{\infty} \sqrt{3} I_{n}\left[\begin{array}{c}
\cos \left[(n-1) \omega_{0} t+\phi_{h p}\right] \\
\sin \left[(n-1) \omega_{0} t+\phi_{h p}\right]
\end{array}\right]
\end{aligned}
$$

The $(6 k \pm 1)$ th $(\mathrm{k}=1,2,3 \ldots)$ harmonic components transform into 6kth $(\mathrm{k}=$ $1,2,3)$ signals in the positive synchronous reference frame. This useful feature simplifies the synchronous frame controller design because only the 6kth $(\mathrm{k}=$ $1,2,3)$ harmonics are compensated.

\subsection{Sliding discrete Fourier transformation}

For a given discrete-time signal x(n), The Discrete Fourier Transformation (DFT) is defined as

$$
\mathrm{X}_{\mathrm{m}}(n)=A_{m} \cos \left(\frac{2 \pi m n}{N}\right)+B_{m} \sin \left(\frac{2 \pi m n}{N}\right)
$$

Where: 


$$
\begin{aligned}
& A_{m}=\frac{2}{N} \sum_{n=0}^{N-1} x(n) \cos \left(\frac{2 \pi m n}{N}\right) \\
& B_{m}=\frac{2}{N} \sum_{n=0}^{N-1} x(n) \sin \left(\frac{2 \pi m \mathrm{n}}{N}\right)
\end{aligned}
$$

DFT often too time-consuming for APF controller requiring a fast dynamic, the sliding DFT (SDFT) method which is computationally simpler than DFT when only part of the frequency spectrum needs to be analyzed, is used to detect harmonics.

When the new real-time data $x(j)$ is sampled and calculated, the old data $\mathrm{x}(\mathrm{j}-\mathrm{N})$ that before a fundamental cycle is substituted by it, so that the new coefficients $A m(j+1)$ and $B m(j+1)$ are obtained.

$$
\begin{aligned}
& A_{m}(\mathrm{j})=A_{m}(\mathrm{j}-1)+\frac{2}{\mathrm{~N}}(x(\mathrm{j})-\mathrm{x}(\mathrm{j}-\mathrm{N})) \cos \left(\frac{2 \pi \mathrm{mn}}{\mathrm{N}}\right) \\
& B_{m}(\mathrm{j})=B_{m}(\mathrm{j}-1)+\frac{2}{\mathrm{~N}}(x(\mathrm{j})-\mathrm{x}(\mathrm{j}-\mathrm{N})) \sin \left(\frac{2 \pi \mathrm{mn}}{\mathrm{N}}\right)
\end{aligned}
$$

With this method, only one subtraction and two multiplications are required for each calculation, and a new calculated value is obtained. This greatly accelerates the updating speed of sampling data and improves the system tracking load current conversion capability and to calculate the accuracy of the reference current. However, when the load current steps, it requires a whole grid fundamental period to track the new load current correctly. Moreover, it requires storing $\mathrm{N}$ sampling points for each phase.

\section{Proposed harmonic dectetion method}

Combining the characteristics of synchronous rotation transform and SDFT, this paper proposes harmonic detection method is shown in Fig. 2. First, thanks to positive synchronous reference frame transformation, load current in synchronous reference frame can be derived as equation (2). It can be concluded all the $(6 k \pm 1)$ th $(\mathrm{k}=1,2,3 \ldots)$ harmonic component transform to $6 \mathrm{kth}(\mathrm{k}=1,2,3 \ldots)$ times grid frequency.

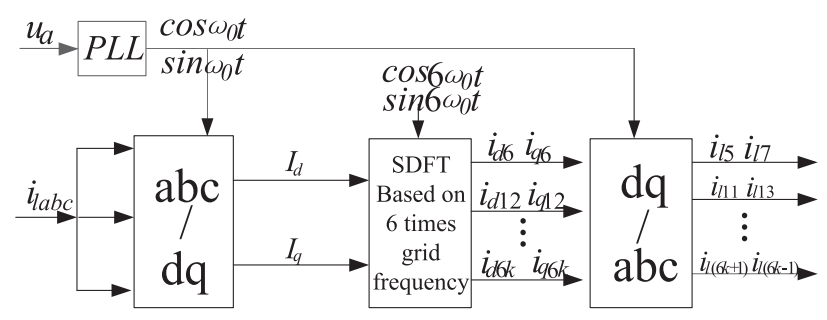

Fig. 2. Proposed harmonics detection method

Then the current reference detection method based on positive synchronous reference frame transform is described as Fig. 2, for the load current has been transformed to $6 \mathrm{k}(\mathrm{k}=1,2,3 \ldots)$ times grid frequency, thus, the SDFT can only detect the $6 \mathrm{k}(\mathrm{k}=1,2,3 \ldots)$ times grid frequency hormonics. In this case, the fundamental frequency of SDFT is determined as 6 times grid frequency would be 
a clever choice, it will get the best dynamic response when the load current steps. $i_{d 6 k}$ and $i_{q 6 k}$ are current reference of d-axis and q-axis respectively.

In the synchronous reference frame, the axis needed to be calculated reduce to 2 , i.e. d-axis and q-axis, compared with 3 of traditional standnary frame, i.e. phase$\mathrm{A}$, phase-B and phase-C. Moreover, because the $(6 k \pm 1)$ th $(\mathrm{k}=1,2,3 \ldots)$ harmonic components in the standnary frame transform to $6 \mathrm{kth}(k=1,2,3 \ldots)$ harmonic components in synchronous reference frame, the numbers of harmonics need to be calculated of each axis reduce to half of traditional standnary frame.

Compared the calculated harmonic number, the storage capacity and the inherent delay with the traditional harmonic detection method and the proposed harmonic detection method, the results are presented in the following table:

Table I. Comparison of the key performance parameters

\begin{tabular}{|c|c|c|}
\hline & traditional method & proposed method \\
\hline $\begin{array}{c}\text { calculated harmonic } \\
\text { number }\end{array}$ & $3 \times 12=36$ & $2 \times 6=12$ \\
\hline memory space occupied & $3 \times N$ & $2 \times \frac{N}{6}=\frac{N}{3}$ \\
\hline inherent delay & $\begin{array}{c}1 \text { fundamental grid } \\
\text { period }\end{array}$ & $\begin{array}{c}\frac{1}{6} \text { fundamental grid } \\
\text { period }\end{array}$ \\
\hline
\end{tabular}

It can be seen that the proposed harmonic detection method has a great advantage, the memory space occupied by the processor will be greatly reduced, which is $\frac{1}{9}$ of the traditional method. At the same time, the calculated harmonic number will be reduced to $\frac{1}{3}$, which makes the number of calculations to achieve greatly reduced. And the rapidity of harmonic detection will be 6 times that of the traditional method, which is helpful to enhance the dynamic response ability of the system.

It means that by using the harmonic detection method proposed in this paper, the system's computing speed, storage space and dynamic response speed are significantly optimized.

Whole APF control system of the proposed strategy is shown as Fig. 3. It incorporates a current feedback control loops, the external dc-link voltage controlling loop aiming to keep the voltage of dc-link capacitor and the voltage feedforward control to prevent grid voltage interference.

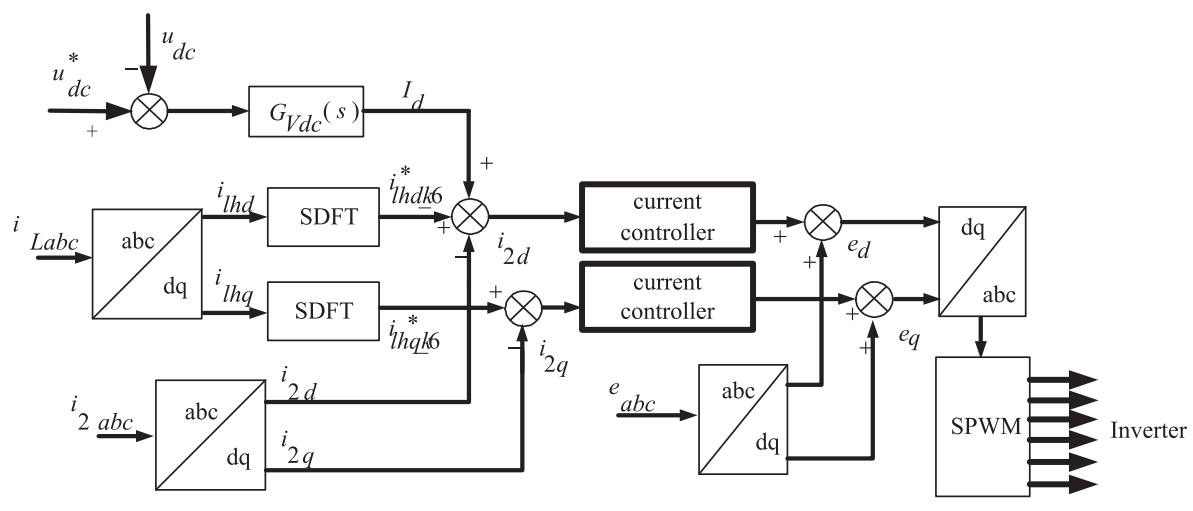

Fig. 3. Overall control of system 


\section{Simulation and experiment result}

To verify the proposed reference current detection method, simulation is made in Matlab/simulink.

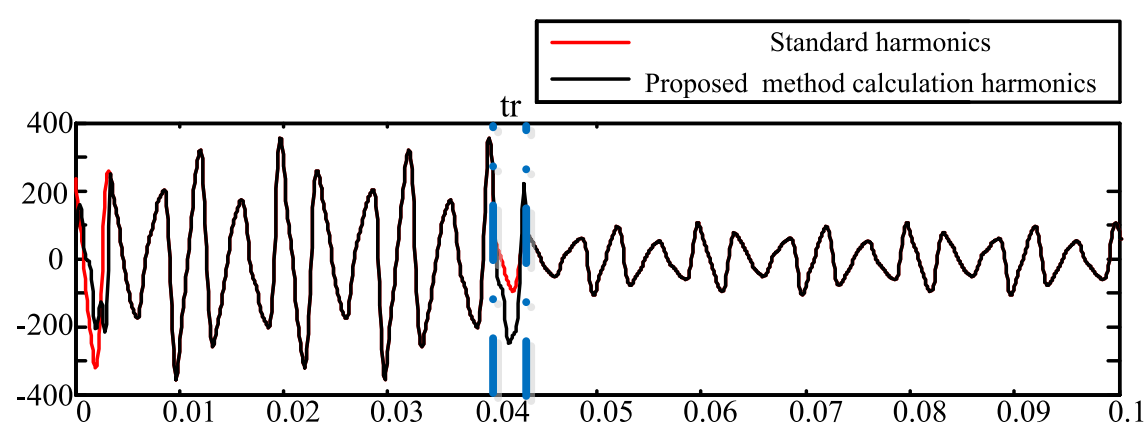

Fig. 4. Simulation results of proposed detection method

In Fig. 4, It can be seen that the proposed harmonics detection method has high accuracy in steady-state calculation and the dynamic adjustment time $t_{r}$ is about $3.3 \mathrm{~ms}$ when the load current steps in $0.04 \mathrm{~s}$. The calculation result is re-accurately tracked. The inherent delay time is reduced to $1 / 6$ fundamental grid period, which is in accordance with the theoretical calculation result and it has quick dynamic response speed.

The system simulation is performed under the following parameters: the switch frequency and sampling frequency of the inverter $12 \mathrm{kHz}$, and the grid frequency is $50 \mathrm{~Hz}$, it means that the $\mathrm{N}$, numbers of sampling point in one fundamental cycle of grid, is 240 . DC-link voltage is set to $750 \mathrm{~V}$; and the APF compensation capacity is $75 \mathrm{~A}$ (rms) for each bridge-arm.

It is observed that the APF with proposed reference current detection method can compensation well for the load harmonic current in Fig. 5(a), and grid current turns to sinusoidal, and the THD of grid current is only $2.53 \%$.

And in the Fig. 5(b) and (c), it illustrates that grid current recovers to sinusoidal in less than half grid fundamental cycle, it is much shorter than that under the traditional SDFT method, which costs approximately two fundamental cycles.
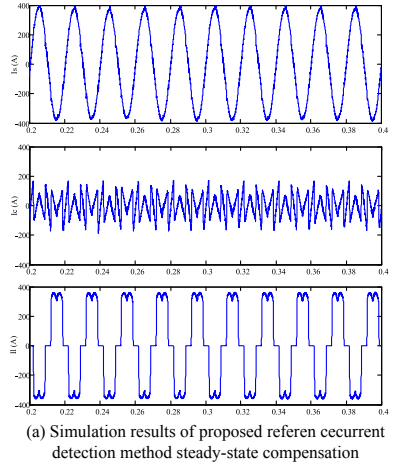

Simulation results of proposed referen cecurre
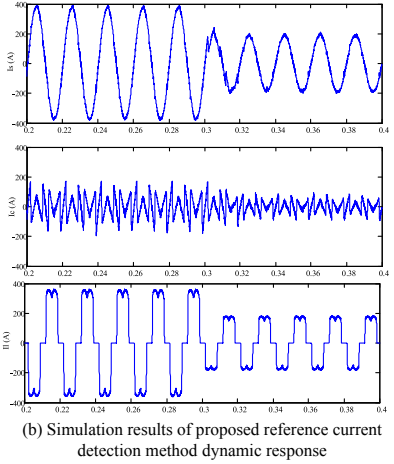
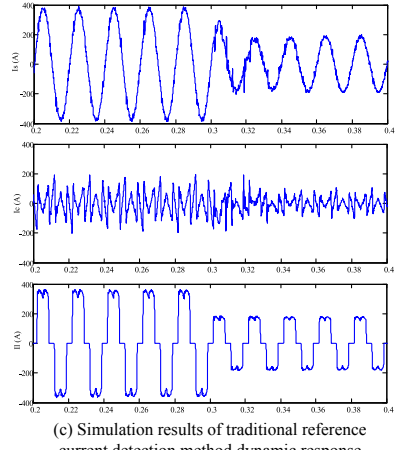

current detection metod dynamic response

Fig. 5. Simulaiton results

To verify the engineering practical value of proposed method, an APF prototype was built, and the main parameters of the APF prototype is the same as the simulation previous. 


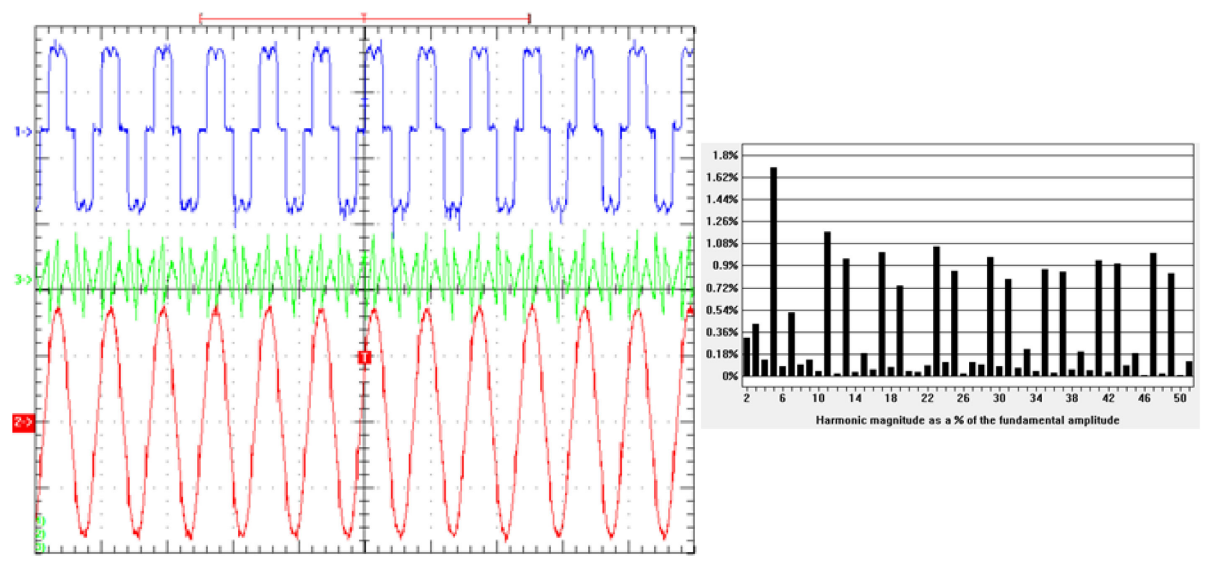

Fig. 6. Experiment results of proposed detection method steady-state compensation

The Fig. 6 shows the waveforms of the grid current of the proposed method is employed. It can be seen when APF steady-state operation, the load harmonic components reaching $28.5 \%$, and the grid current achieve the desired sinusoidal after compensation, the THD is only $3.87 \%$.

The experiment results of the traditional SDFT reference current detection method and the fast reference current detection methods, which presented in this paper, dynamic response comparison is shown in Fig. 7.

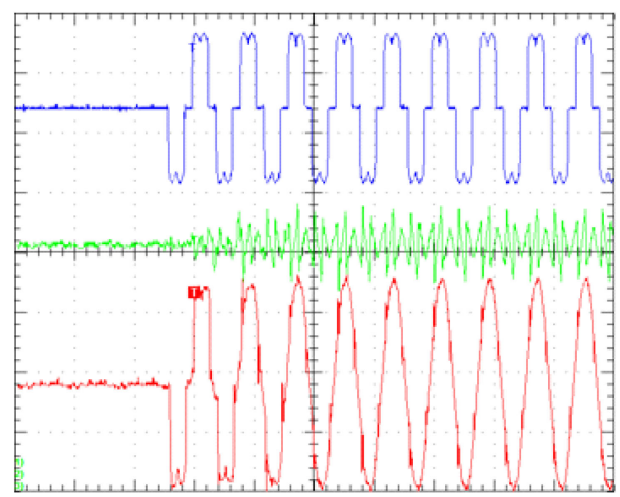
detection method dynamic response (a) Experiment results of traditional SDFT harmonics

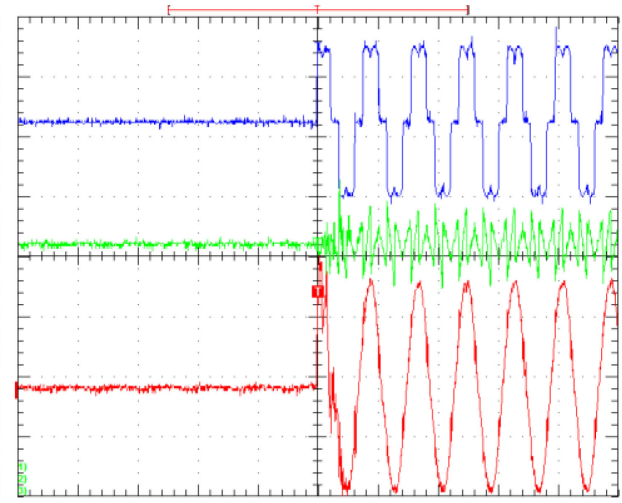

(b) Experiment results of proposed harmonics detection method dynamic response
Fig. 7. Experiment results of dynamic response

It indicates that the experimental results are similar to the simulation results. The grid current recovers to sinusoidal in less than half grid fundamental cycles, it is much shorter than that under the traditional SDFT method which costs approximately two fundamental cycles.

\section{Conclusion}

In this paper, an effective refrence current detection method based on synchronous reference frame and SDFT for the shunt APF is presented. Firstly sampled load current signals were transformed from abc-frame to dq-frame then the signals were transformed from time-domain into frequency-domain by using SDFT, and trans- 
formed the extraction harmonics back to time domain under dq-frame, by this way, the concerned parts as reference current are inverted into abc-frame. Finally, simulation and experiment results verify the proposed method is valid and has fast response speed. 